\title{
A review on economically adsorbents on heavy metals removal in water and wastewater
}

\begin{abstract}
Heavy metals contamination in water has been an issue to the environment and human health. The persisting contamination level has been observed and concerned by the public due to continuous deterioration of water quality. On the other hand, conventional treatment system could not completely remove the toxic metals in the water, thus alternative purification methods using inexpensive materials were endeavor to improve the current treatment process. Wide ranges of low cost adsorbents were used to remove heavy metal in aqueous solution and wastewater. The low cost adsorbents were usually collected from agricultural waste, seafood waste, food waste, industrial by-product and soil. These adsorbents are readily available in a copious amount. Besides, the pretreatment are not complicated to be conducted on the raw products, which is economically sound for an alternative treatment. The previous studies have provided much evidence of low cost adsorbents' efficiency in removing metal ions from aqueous solution or wastewater. In this review, several low cost adsorbents in the recent literature have been studied. The maximum adsorption capacity, affecting factors such as $\mathrm{pH}$, contact times, temperature, initial concentration and modified materials were revised and summarized in this review for further reference. Comparisons of the adsorbent between the modified and natural products were also demonstrated to provide a clear understanding on the kinetic uptake of the selected adsorbents. Some of the natural adsorbents appeared as good heavy metal removal, while some were not and require further modifications and improvements to enhance the adsorption capacity. SWOT analysis (strength, weakness, opportunities, threat) was also performed on the low cost adsorbents to identify the advantages of using low cost adsorbents and solve the weaknesses encountered by the utilization of low cost materials. This tool helps to determine the potential quality of low cost materials in the application for water and wastewater treatment.
\end{abstract}

Keyword: Low cost; Adsorbents; Heavy metals; Adsorption capacity; SWOT analysis 